\title{
FIRST ASSESSMENT OF THE PERMANENT SCATTERER LINEAR DISPLACEMENT MODEL IN AIRBORNE INSAR TIME SERIES
}

\author{
Karlus A. Câmara de Macedo
}

OrbiSat Remote Sensing

Av. Shishima Hifumi, 2911

São José dos Campos - SP, Brazil

karlus.macedo@orbisat.com.br

\author{
Rolf Scheiber, Alberto Moreira
}

\author{
German Aerospace Center \\ Microwaves and Radar Institute \\ Wessling, Germany \\ (rolf.scheiber;alberto.moreira)@dlr.de
}

\begin{abstract}
This paper presents the very first assessment of the permanent scatterer (PS) technique for airborne data. A data set of 14 SAR images at L-band, acquired over the Oberpfaffenhofen area on the same day with the E-SAR system of the German Aerospace Center (DLR), is used for the first airborne time series analysis with PS. The paper shows the importance of mitigating the residual motion errors through the use of precise motion compensation strategy before PS analysis. The target velocity and DEM error results are obtained by a periodogram-based estimation considering the linear displacement model. Due to the small number of images in our data set, the displacement velocity and DEM error results are presented on a PS basis. Target structures related to selected reliable PSs are shown and the corresponding periodograms highlighted.
\end{abstract}

Index Terms - airborne SAR, permanent scatterers, linear displacement model, residual motion errors

\section{INTRODUCTION}

The permanent scatterer (PS) technique is a well-established advanced D-InSAR method that has proven to have a remarkable potential for mapping ground deformations [1]. By performing a time series analysis on a SAR data set of 30 or more images, a dense grid of phase-stable scatterers (known as permanent scatterers) can be identified. Estimation using the selected PSs can achieve a precision for ground deformation measurements in the order of $1 \mathrm{~mm}$ for C-band spaceborne SAR over urban areas [2]. Another advanced D-InSAR method often used for ground deformation is the small baseline technique (SB) [3, 4].

For spaceborne images, the advanced D-InSAR techniques are able to deliver reliable results while airborne D-InSAR is still a difficult task. This is due to the lack of time-periodic data available along the years, and due to the instable trajectory described by the airborne platforms, demanding the use of a precise Motion Compensation (MoComp) technique $[5,6,7]$. Several airborne experiments and campaigns have been carried out using the classical 2or 3-pass D-InSAR [8, 9, 10]. For estimation of the surface velocity field of glaciers moving $5 \mathrm{~cm} /$ day, the 3 -pass airborne D-InSAR is able to deliver realiable results after topographydependent MoComp and residual phase estimation [11]. For displacements measurements with global accuracy in the order of millimeters, no validated results were achieved up to now with airborne systems. The use of time series analysis can help airborne D-InSAR to become reliable and accurate as in the spaceborne case. Airborne D-InSAR is an interesting tool for mapping ground deformation due to its flexibility compared to the spaceborne case, enabling for example flexible deployment with the desired revisit time.

Analysing the archive of data acquired with the E-SAR system of DLR from 1998 until 2005, one set of 14 airborne SAR images at L-band, acquired over the Oberpfaffenhofen area on the same day, was identified as feasible for a first airborne time series analysis. In 2006, a first airborne PS selection with this data set was published, where a trade-off between the number of images, the desired phase stability and the detectability of the permanent scatterers was shown [12].

Now, with the same 14-image data set, we perform for the first time a complete time series analysis with the selected PS in order to assess the periodogram-based estimation of the linear displacements and DEM (Digital Elevation Model) errors. Due to the small number of images in our data set, the final number of reliable PS is not enough to form a dense grid of estimations to enable an analysis of a wide area. Therefore, the displacement velocity and DEM error results are presented on a PS basis. Target structures related to selected reliable PSs are shown and the corresponding periodograms are highlighted. Recently a time series analysis with airborne data applying the SB technique was published [13] using the same data set.

In this paper we analyse the PS results before and after applying a complete motion compensation strategy with the Precise Topography- and Aperture-dependent (PTA) MoComp and the Weighted Phase Curvature Autofocus (WPCA) algorithms in order to mitigate phase errors due to flight devi- 
ations from the nominal track [7, 14]. Specifically, the paper shows the increase in the number of PS candidates and the increase of the coherence between the model and the interferometric phase after applying a complete MoComp strategy. The paper concludes that the phase undulations should be mitigated as much as possible before applying time series analysis (residual terms should be less or equal than 0.6 radians [2]).

This paper is organized as follows. Section 2 shows the selected PS candidates and how they are related to the use of precise MoComp techniques during SAR processing. Section 3 analyses the stack of differential interferograms obtained with and without the use of precise MoComp techniques. Section 4 presents the results of displacement velocity and DEM error estimations on a PS basis. Section 5 addresses the conclusions.

\section{PS CANDIDATE SELECTION}

In order to perform the PS detection, all slave images were accurately coregistered and calibrated relative to the master image. The data set is composed of 14 images at L-band (HH) acquired over the Oberpfaffenhofen area on May 11, 1998, within 143 minutes (every 10 minutes) and with baseline lengths going from 7 to 122 meters.

The PS selection is performed by setting the dispersion index threshold $D_{T}$ to 0.06 (due to the low number of available images) to allow a low false alarm rate $P_{f a}$ of PSs with dispersion equal or less than 0.25 as shown in [12]. With $D_{T}=0.06$, we have $P_{f a}=10^{-5}$, but we pay the price of having a detection probability of only $17 \%$. Fig. 1 shows the amplitude images and the selected PSs painted in red. Six out of eleven corner reflectors are selected as PS as indicated by the white circles and labeled with CR.

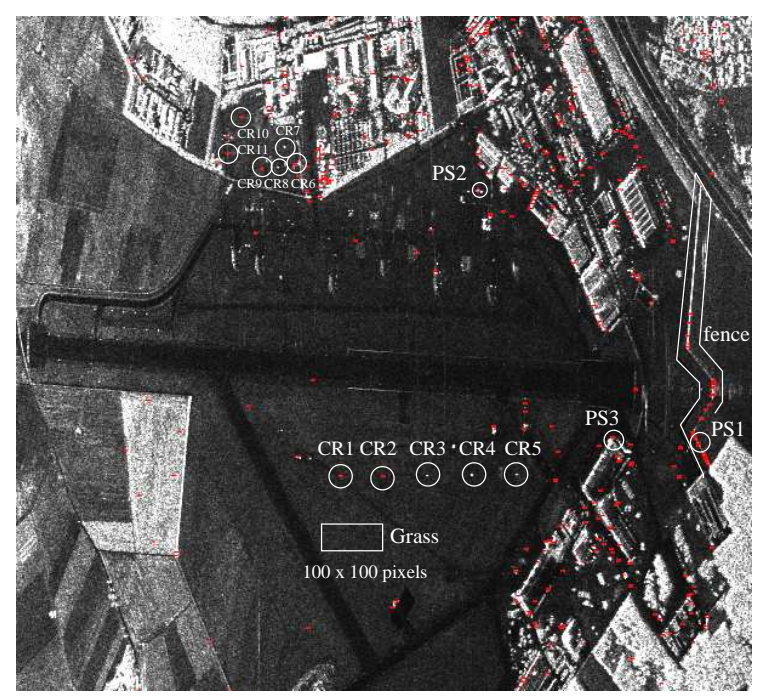

Fig. 1. Amplitude image and PS candidates (in red)
The SAR processing chain applied to the data set involves the ECS (Extended Chirp Scaling) integrated with MoComp to a reference level [15], followed by the use of Precise Topography- and Aperture-dependent MoComp (PTA) and the Weighted Phase Curvature Autofocus (WPCA) [7, 14]. For analysis purposes, the PS selection was performed in the images processed with and without applying the complete motion compensation strategy, PTA-WPCA.

Table 1 shows the number of permanent scatterer candidates with $D_{T}=0.06$. As expected, the number of PSs increases as the residual phase errors are mitigated. This increase is directly related to a better impulse response function (IRF) focusing and positioning achieved after PTA-WPCA processing. The better the focusing and the positioning, the more stable becomes the phase of the scatterer leading to a greater number of detectable PSs.

Table 1. Number of PS candidates (14 images, $D_{T}=0.06$ )

\begin{tabular}{|l|c|c|c|}
\hline & no PTA-WPCA & after PTA & after PTA-WPCA \\
\hline PSs & 361 & 426 & 551 \\
\hline
\end{tabular}

\section{DIFFERENTIAL INTERFEROGRAMS}

With 14 images, 13 differential (residual) interferograms are available for terrain deformation and DEM error estimations through PS time series analysis. According to [2], the residual phase errors, which come mainly from atmospheric effects in the spaceborne case, may not exceed 0.6 radians in order to properly model the terrain deformation and DEM errors. In the airborne case, by analogy, the residual phase errors, which come mainly from deviations from the nominal track, have to be compensated. Thus, for comparison purposes, the airborne interferograms are analysed before and after applying the PTA-WPCA processing.

Fig. 3(a) shows the residual interferogram (number 1) obtained by performing typical SAR processing, i.e. ECS integrated with MoComp to a reference level. Fig. 3(b) shows the same interferogram after PTA-MoComp and Fig. 3(c) shows it after PTA-WPCA. Finally, Fig. 3(d) shows the differential interferogram, after PTA-WPCA and removal of the offset and linear global terms, to be used effectively with the stack of 13 interferograms for periodogram-based PS estimations. The other 12 inteferograms are presented in [16]. In the next section of this paper, we perform the periodogram-based PS estimations for two cases, "no residual MoComp" and "after PTA-WPCA", both with linear global terms removal.

\section{LINEAR DISPLACEMENT AND DEM ERROR}

With the differential phases available, the displacement velocity and the DEM error at the PS candidates are estimated 


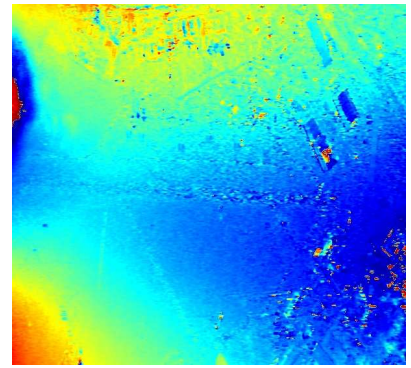

(a) no residual MoComp

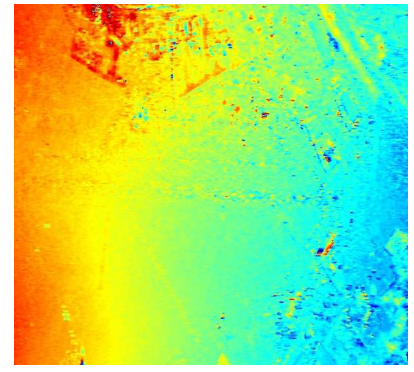

(c) after PTA-WPCA

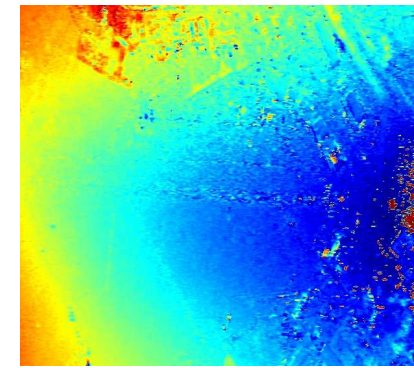

(b) after PTA

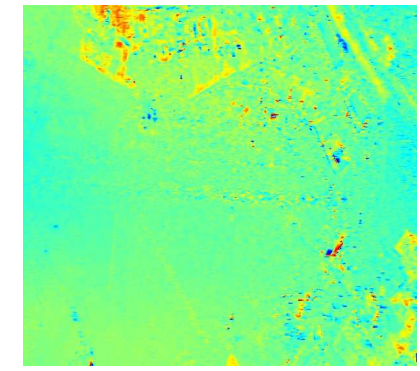

(d) linear global terms removed

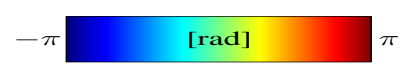

Fig. 2. A residual interferogram (no.1) out of the stack of 13

by maximizing the following periodogram,

$$
\Upsilon=\frac{1}{N}\left|\sum_{n=0}^{N} e^{\left(j \psi_{\text {diff }, n}-j \frac{4 \pi}{\lambda}\left(t_{n} v+\frac{B_{n} \cos \left(\theta-\alpha_{n}\right)}{r \sin \theta} \varepsilon_{\text {topo }}\right)\right)}\right|
$$

where $\psi_{\text {diff, } n}$ are the observed differential phases, $v$ is the displacement velocity, $\varepsilon_{\text {topo }}$ is the DEM error, $\theta$ is the local look angle, $B_{n}$ and $\alpha_{n}$ are the baseline lengths and the tilt angles of the individual interferometric pairs, $\lambda$ is the wavelength, and $\Upsilon$ corresponds to the coherence between the model and the observed phases. A value of $\Upsilon=1$ corresponds to total correlation with the model and 0 to total decorrelation.

A reliable deformation velocity and the DEM error are obtained by thresholding $\Upsilon$. For 25 SAR images, the coherence threshold $\Upsilon_{T}$ shall be set to 0.8 in order to have $1 \%$ of outliers, i.e. observations that are statistically distant from the rest of the data. For 15 images, the $\Upsilon_{T}$ shall be set to 0.98 for $1 \%$ of outliers according to [2].

If we choose $\Upsilon_{T}=0.9$ for this 14-image data set, we obtain 446 PSs (out of 551 candidates selected after PTA-WPCA), where 42 PSs are modeled as moving with a rate of -3.45 $\mathrm{mm} / \mathrm{h}$ (millimeters per hour). These 42 , i.e. about $10 \%$ of the 446 are very likely outliers, because we have chosen a threshold of less then 0.98 . If we choose $\Upsilon_{T}=0.98$ for this 14-image data set, we obtain 9 reliable PSs (out 361 candidates selected before PTA-WPCA) and 51 reliable PSs (out of 551 candidates selected after PTA-WPCA). In both cases the PSs do not move at all. For a fair comparison with the motion compensated time series data set, the constant and linear global terms of residual motion errors were removed in both cases, before and after residual MoComp.

Fig. 3 shows the periodograms before and after PTAWPCA for 2 different reliable PSs, PS1 (fence) and PS2 (container), as indicated by white circles in Fig. 1 and zoomed and depictured in Fig. 3. It is clear to see that after PTAWPCA processing the peak of the periodogram gets closer to 1 , showing a better coherence with the model, which is directly related to the mitigation of residual motion errors.

Table 2 and Table 3 show the estimation results of 4 PSs, including one (a container) that before PTA-WPCA had $\Upsilon_{T}=0.91$ and was mistakenly modeled as moving. After PTA-WPCA its coherence became equal to 0.98 and its differential phase was correctly modeled as DEM error and not as displacement. The standard deviation of the estimations are computed according to [1].

Table 2. DEM error estimations at single PSs

\begin{tabular}{|c|c|c|}
\hline & no PTA-WPCA & with PTA-WPCA \\
\hline PS & $\varepsilon_{\text {topo }} \pm \sigma_{\varepsilon}[\mathrm{cm}]$ & $\varepsilon_{\text {topo }} \pm \sigma_{\varepsilon}[\mathrm{cm}]$ \\
\hline fence (PS1) & $-110 \pm 16.9$ & $-90.0 \pm 8.28$ \\
container (PS2) & $0.00 \pm 16.1$ & $10.0 \pm 5.36$ \\
building (PS3) & $-30.0 \pm 15.1$ & $-10.0 \pm 6.74$ \\
corner (CR1) & $-10.0 \pm 5.56$ & $-10.0 \pm 2.76$ \\
\hline
\end{tabular}

Table 3. Displacement estimations at single PSs

\begin{tabular}{|c|c|c|}
\hline & no PTA-WPCA & with PTA-WPCA \\
\hline PS & $v \pm \sigma_{v}[\mathrm{~mm} / \mathrm{h}]$ & $v \pm \sigma_{v}[\mathrm{~mm} / \mathrm{h}]$ \\
\hline fence (PS1) & $0.000 \pm 0.028$ & $0.000 \pm 0.014$ \\
container (PS2) & $-3.451 \pm 0.037$ & $0.000 \pm 0.012$ \\
building (PS3) & $0.000 \pm 0.029$ & $0.000 \pm 0.013$ \\
corner (CR1) & $0.000 \pm 0.016$ & $0.000 \pm 0.008$ \\
\hline
\end{tabular}

\section{CONCLUSIONS}

The use of multiple acquisitions allowed for the first time reliable airborne D-InSAR measurements with millimeter accuracy. The observed differential phases were modeled as DEM errors and no movements were detected. This is expected due to the short-time span and because the DEM was derived from the E-SAR X-band (VV) InSAR system, while the 14 images were acquired at L-band (HH). The DEM errors can be related to the different phase centers of the scatterer, carrier frequencies, polarizations and/or interpolation used to back-geocode the X-band data into the L-band acquisition geometry.

It can been seen that if the periodogram's threshold is not correctly set and/or the motion errors are not well compensated, DEM errors can be wrongly modeled as displacement. Furthermore, mitigation of motion errors increases the number of permanent scatterers available for D-InSAR analysis.

For a complete analysis of DEM errors and terrain deformations of the scene, a high density of PS is required. Further work will focus on the work with a data set made of a larger number of images over urban areas. 

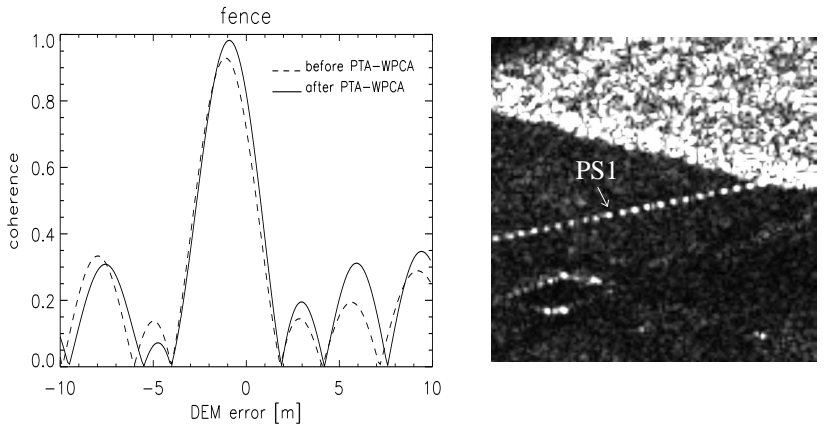

(a) DEM error, fence

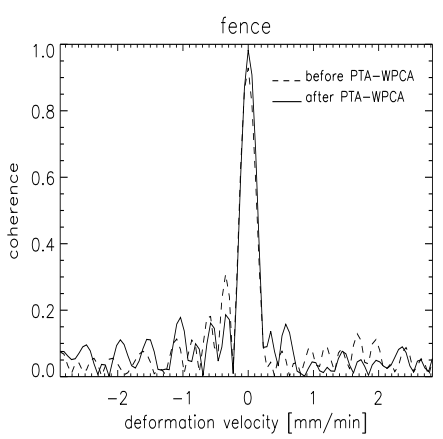

(c) velocity, fence

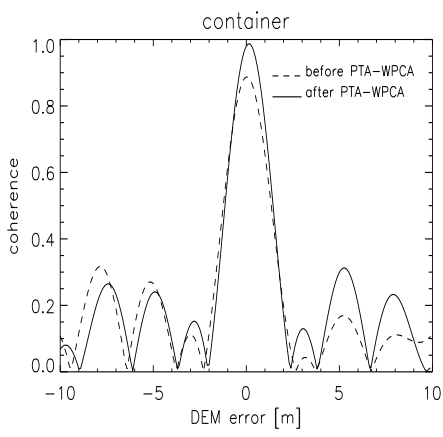

(e) DEM error, container

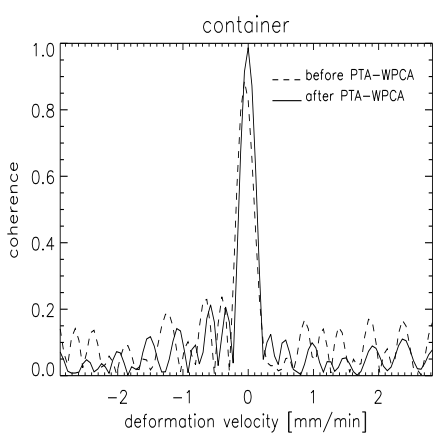

(g) velocity, container (b) SAR data, fence

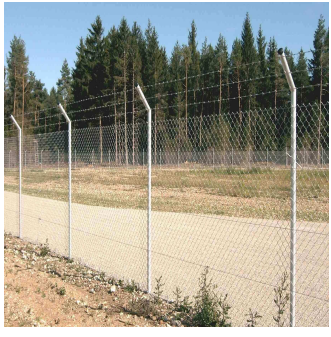

(d) photo, fence

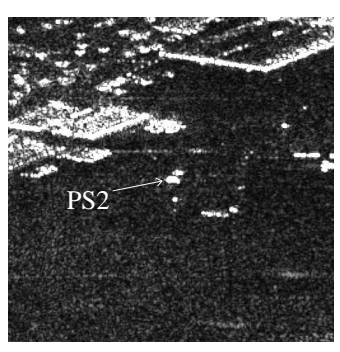

(f) SAR data, container

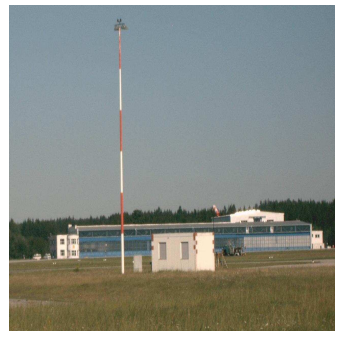

(h) photo, container

Fig. 3. Periodogram of two selected reliable PSs and their related data

\section{REFERENCES}

[1] A. Ferretti, C. Prati, and F. Rocca, "Permanent scatterers in SAR interferometry," IEEE Trans. Geosci. Remote Sens., vol. 39, no. 1, pp. 8-20, Jan. 2001.

[2] C. Colesanti, A. Ferretti, F. Novali, C. Prati, and F. Rocca, "SAR monitoring of progressive and seasonal ground deformation using the permanent scatterer technique," IEEE Trans. Geosci. Remote Sens., vol. 41, no. 7, pp. 1685-1701, Jul. 2003.

[3] P. Berardino, G. Fornaro, R. Lanari, and E. Sansosti, "A new algorithm for surface deformation monitoring based on small baseline differential SAR interferograms," IEEE Trans. Geosci. Remote Sens., vol. 40, no. 11, pp. 2375-2383, Nov. 2002.

[4] R. Lanari, O. Mora, M. Manunta, J. J. Mallorqui, P. Berardino, and E. Sansosti, "A small-baseline approach for investigating deformations on full-resolution differential SAR interferograms," IEEE Trans. Geosci. Remote Sens., vol. 42, no. 7, pp. 1377 - 1386, Jul. 2004.

[5] A. Reigber, P. Prats, and J.J. Mallorqui, "Refined estimation of timevarying baseline errors in airborne SAR interferometry," IEEE Geosci. Remote Sens. Lett., vol. 3, no. 1, pp. 145-149, Jan. 2006.

[6] P. Prats, K.A.C. de Macedo, A. Reigber, R. Scheiber, and J.J. Mallorqui, "Comparison of topography- and aperture- dependent motion compensation algorithms for airborne SAR," IEEE Geosci. Remote Sens. Lett., vol. 4, no. 3, pp. 349-353, Jul. 2007.

[7] K.A.C. de Macedo, R. Scheiber, and A. Moreira, "An autofocus approach for residual motion errors with application to airborne repeatpass SAR intertferometry," IEEE Trans. Geosci. Remote Sens., vol. 46, no. 10, pp. 3151-3162, Oct. 2008.

[8] A. Reigber and R. Scheiber, "Airborne differential SAR interferometry: First results at L-band," IEEE Trans. Geosci. Remote Sens., vol. 41, no. 6, pp. 1516-1520, Jun. 2003.

[9] K.A.C. de Macedo, C. Andres, and R. Scheiber, "On the requirements of SAR processing for airborne differential interferometry," Proc. IGARSS'05: IEEE Intl. Geosci. Remote Sens. Symposium, Seoul, South Korea, Jul. 2005.

[10] S. Perna, C. Wimmer, J.R. Moreira, and G. Fornaro, "X-band airborne differential interferometry: Results of the OrbiSAR campaign over the Perugia area," IEEE Trans. Geosci. Remote Sens., vol. 46, no. 2, pp. 489-503, Feb. 2008.

[11] P. Prats, R. Scheiber, A. Reigber, C. Andres, and R. Horn, "Estimation of the surface velocity field of the Aletsch glacier using multibaseline airborne SAR interferometry," IEEE Trans. Geosci. Remote Sens., vol. 47, no. 2, pp. 419 - 430, Fev. 2009.

[12] K.A.C de Macedo, R. Scheiber, and A. Moreira, "First evaluations of airbone InSAR time-series," Proc. EUSAR'06: European Conference on Synthetic Aperture Radar, Dresden, Germany, May 2006.

[13] P. Prats, A. Reigber, J. J. Mallorqui, R. Scheiber, and A. Moreira, "Estimation of the temporal evolution of the deformation using airborne differential SAR interferometry," IEEE Trans. Geosci. Remote Sens. vol. 46, no. 4, pp. 1065-1078, Apr. 2008.

[14] K.A.C. de Macedo and R. Scheiber, "Precise topography- and aperture-dependent motion compensation for airborne SAR," IEEE Geosci. Remote Sens. Lett., vol. 2, no. 2, pp. 172-176, Apr. 2005.

[15] A. Moreira and Y. Huang, "Airborne SAR processing of highly squinted data using a chirp scaling approach with integrated motion compensation," IEEE Trans. Geosci. Remote Sens., vol. 32, no. 5, pp. 1029-1040, Sept. 1994.

[16] K. A. C. de Macedo, New processing methodology for airborne repeat-pass SAR interferometry, Ph.D. thesis, Department of Eletrical Engineering and Information Technology, University of Karlsruhe, Germany, 2008. 